\title{
Is resistance to novobiocin a reliable test for confirmation of the identification of Staphylococcus saprophyticus?
}

\author{
LESLEY A. McTAGGART and T.S. J. ELLIOTT \\ Department of Clinical Microbiology, Queen Elizabeth Hospital, Edgbaston, Birmingham B15 2TH
}

\begin{abstract}
Summary. Staphylococcus saprophyticus, a coagulase-negative staphylococcus (CNS), causes acute urinary tract infection predominantly in young women (15-30 years). In the clinical microbiology laboratory identification and differentiation of $S$. saprophyticus from other CNS usually depends solely upon the demonstration of resistance to the antimicrobial agent novobiocin. Phenotypic characteristics of 36 novobiocinresistant CNS isolated from the urine of patients with acute urinary tract infections were further analysed and the homogeneity of the isolates assessed. The organisms were speciated by the API STAPH identification system. Twenty-one isolates were S. saprophyticus $(\mathrm{p} \geq 97 \%)$, and there was one strain each of $S$. epidermidis, $S$. hominis and $S$. simulans $(\mathrm{p} \geq 97 \%)$. Of the remainder, three isolates were unidentifiable and a further nine had the characteristics associated with more than one species of CNS. Additional tests, including carbohydrate fermentation, antibiotic sensitivity and fluorogenic substrate utilisation, were performed on all isolates. Computer analysis of the results confirmed that testing for resistance to novobiocin selects a heterogeneous group of CNS composed of several different species.
\end{abstract}

\section{Introduction}

Coagulase-negative staphylococci (CNS), under certain conditions, may cause various infections that include intravascular-catheter-associated septicaemia, endocarditis and urinary tract infection (UTI).

In 1962, Torres Pereira isolated from urine a group of CNS that shared several phenotypic characteristics, including resistance to novobiocin at a concentration of $5 \mu \mathrm{g} / \mathrm{ml}$. It was postulated that these CNS represented a newly recognised urinary tract pathogen. Maskell (1974) subsequently confirmed that a particular species of CNS, Staphylococcus saprophyticus, had a pathogenic role in community-acquired UTI, predominantly in young women.

The recognition of $S$. saprophyticus as a pathogen resulted in the requirement for CNS isolates from UTI to be identified to species level. Kloos and Schleifer (1975) had previously described a scheme for the identification and speciation of the genus Staphylococcus. The scheme involved the use of a variety of media and reagents and a requirement to incubate the organisms for prolonged periods. The

Received 5 June 1989; accepted 22 June 1989 scheme was, therefore, unsuitable for routine clinical laboratories. Several abbreviated and rapid schemes for the identification of staphylococci have been developed subsequently (Brun et al., 1978; Christensen et al., 1983; Morgan, 1983; Bes et al., 1985; Hussain et al., 1986). These methods incorporated resistance to novobiocin at a concentration of $5 \mu \mathrm{g} / \mathrm{ml}$ for the presumptive identification of $S$. saprophyticus. However, it has now been recognised that other CNS are also resistant to novobiocin at this concentration, including $S$. cohnii, $S$. sciuri and S. xylosus (Hussain et al., 1986).

In the present investigation, strains of presumptive $S$. saprophyticus, identified by novobiocin resistance, which had been isolated from patients with acute UTI, were examined further to evaluate the selectivity of this test. An extended range of identification tests was used not only to determine the homogeneity of this group of organisms but also to investigate the value of other potential methods for the identification of $S$. saprophyticus.

\section{Materials and methods}

\section{Organisms}

Thirty-six isolates of presumptive $S$. saprophyticus were obtained from the urine of patients with acute UTI. All 
the organisms were present at a concentration of $\geq 10^{5} / \mathrm{ml}$ of urine and were considered to be clinically significant. The CNS were identified by colonial appearance, Gram's stain, positive catalase reaction and negative coagulase and deoxyribonuclease (DNAase) reactions ( $\mathrm{Yu}$ and Washington, 1985). A presumptive identification of $S$. saprophyticus was derived from resistance to novobiocin. This was performed by inoculation of DST agar (Oxoid) containing novobiocin (Sigma) $5 \mu \mathrm{g} / \mathrm{ml}$. The presumptive $S$. saprophyticus isolates were further tested by the API STAPH biochemical strip (API Systems, Manuvix, France) to confirm the species identification.

Representative strains of other staphylococcal species described by Nachaie (1983) were kindly supplied by Dr M. Goodfellow, University of Newcastle upon Tyne. These strains had been isolated from human and veterinary sources worldwide. The isolates were included in the subsequent investigations for comparisons with the presumptive $S$. saprophyticus strains.

\section{Carbohydrate fermentation tests}

The staphylococci were tested for the ability to ferment carbohydrates (table I) by the replicator method described by Lennox and Ackerman (1984).

\section{Antibiotic sensitivity tests}

Antibiotic sensitivities of the staphylococcal isolates were determined by a replicator method. The isolates were multipoint inoculated on to DST Agar (Oxoid) plates containing one of the following antibiotics: chloramphenicol $10 \mu \mathrm{g} / \mathrm{ml}$, erythromycin $2 \mu \mathrm{g} / \mathrm{ml}$, gentamicin $4 \mu \mathrm{g} / \mathrm{ml}$, neomycin $10 \mu \mathrm{g} / \mathrm{ml}$, netilmicin $4 \mu \mathrm{g} / \mathrm{ml}$, novobiocin $5 \mu \mathrm{g} / \mathrm{ml}$, penicillin $0 \cdot 1 \mu \mathrm{g} / \mathrm{ml}$, tobramycin $4 \mu \mathrm{g} / \mathrm{ml}$, tetracycline $2 \mu \mathrm{g} / \mathrm{ml}$, or trimethoprim $1.25 \mu \mathrm{g} /$ $\mathrm{ml}$. Resistance was determined by growth of the isolate on the medium containing antibiotic.

The bacteria were also inoculated on to Phenolphthalein Phosphate Agar (Oxoid) to determine phosphatase activity.

Table I. Carbohydrates utilised in fermentation tests. The staphylococci were inoculated on to agar plates containing individual carbohydrates at a concentration of $20 \mathrm{~g} / \mathrm{L}$. Fermentation was determined by $\mathrm{pH}$ change as described by Lennox and Ackerman (1984)

\begin{tabular}{lll}
$\mathrm{L}+$ arabinose & Me- $\beta$-D-glucopyranoside & rhamnose \\
cellobiose & inositol & sorbitol \\
$\beta$-D-fructose & lactose & sucrose \\
galactose & maltose & trehalose \\
$\beta$-D-gentobiose & mannitol & turanose \\
glucose & mannose & xylitol \\
Me- $\alpha$-D-glucopyranoside & D-raffinose & xylose \\
\hline
\end{tabular}

\section{Enzyme profile}

Various enzyme activities of the staphylococcal strains were determined. Non-fluorogenic derivatives of 7amino-4-methylumbelliferone and 7-amino-4-methylcoumarin (tables II and III) were used to detect the presence of a series of enzymes. The derivatives consisted of various amino acids, organic and inorganic esters and glycosides bound to a methylumbelliferone or methylcoumarin marker. When specific enzymes were present which could degrade the covalent bond the fluorogenic moiety was released.

To determine the presence of specific enzymes the organisms were inoculated on to $5 \%$ horse blood agar (Difco) and incubated at $37^{\circ} \mathrm{C}$ for $18 \mathrm{~h}$. A bacterial colony was then removed and emulsified with $20 \mu \mathrm{l}$ of the fluorogenic substrate on Whatman's grade 1 filter paper which was incubated for 30 minutes at $37^{\circ} \mathrm{C}$. The activity of an enzyme which had degraded carbohydrate was determined by the appearance of fluorescence when exposed to UV light.

Table II. The derivatives of 7-amino-4-methylumbelliferone used in the survey of phenotypic characteristics of staphylococci

\section{Organic esters : \\ oleate \\ palmitate \\ stearate}

\section{Glycosides:}

2-acetamido-2-deoxyD-glucopyranoside $\alpha$-L-arabinofuranoside $\beta$-L-arabinofuranoside $\beta$-D-cellobiopyranoside $\beta$-D-fucopyranoside $\alpha$-D-galactopyranoside $\beta$-D-galactopyranoside

\author{
Inorganic esters : \\ bis-phosphate \\ phosphate (acid) \\ phosphate (alkali) \\ pyrophosphate \\ sulphate
}

\author{
$\alpha$-D-glucopyranoside \\ $\beta$-D-glucopyranoside \\ $\beta$-D-mannopyranoside \\ $\beta$-D-xyloside \\ $\mathrm{N}_{1} \mathbf{N}^{1}$-Diacetylchitobiose \\ $2^{1}-4$ meu-K-D- $N$ \\ acetylneuramic acid
}

Table III. The amino acid derivatives of 7-amino-4methylcoumarin (AMC) used in the survey of phenotypic characteristics of isolates of staphylococci

L-ala-AMC

H-ala-AMC

H-ala-ala-phe-AMC. $\mathrm{HCl}$

Z-arg-arg-AMC. $2 \mathrm{HCl}$

N-cbz-L-arg-AMC. $\mathrm{HCl}$

$\mathrm{H}$-asp-AMC.OH

H-glu-AMC.OH

gly-AMC.HBr

Z-gly-gly-leu-AMC

Z-gly-pro-AMC

H-lys-AMC 2 acetate $1 \mathrm{AcOH}$
get-get-gly-phe-AMC

H-met-AMC.AcOH

H-orn-AMC

pyr-AMC

H-ser-AMC.AcOH

H-thr-AMC

H-tyr-AMC

L-val-AMC

Z-phe-arg-AMC

boc-val-pro-arg-AMC 


\section{Computer analysis}

The results of the antibiotic sensitivities, carbohydrate fermentations and enzyme profile tests were analysed with the Clustan programme developed by Sneath (1957). The programme produced a dendrogram in which organisms with similar result profiles were grouped together.

The percentage of positive and negative results for each test carried out on the individual strains of staphylococci were determined. The species specificity of each test was then assessed.

\section{Results}

The 36 clinical isolates of presumptive $S$. saprophyticus identified by novobiocin resistance were further examined by the API STAPH biochemical strip. Data base analysis of the results confirmed that 21 isolates were $S$. saprophyticus $(\mathrm{p} \geq 97 \%)$. Three of the isolates tested were unidentifiable. A further nine strains had characteristics attributable to more than one species of CNS and were given an indeterminate identification. The remaining three isolates were positively identified as $S$. epidermidis, $S$. hominis or $S$. simulans $(\mathrm{p} \geq 97 \%$ ).

Together with representative strains of other staphylococcal species the 36 clinical isolates were examined for the ability to ferment certain carbohydrates (tables IV and V), sensitivity to antibiotics (tables VI and VII) and for the capacity to utilise fluorogenic substrates (tables VIII, IX, X and XI). From these results, the organisms were subsequently grouped by Clustan analysis (figure) into 34 clusters (table XII) containing organisms with common phenotypic characteristics. Of the original 36 clinical isolates examined, 28 strains were allocated to six different clusters. Included within these six clusters were one strain of $S$. cohnii or $S$. equorum, and two strains of $S$. xylosus.

The remaining eight clinical isolates of presumptive $S$. saprophyticus were distributed throughout the other clusters. One isolate was grouped with three strains of $S$. simulans which were derived from the genito-urinary tract of patients. Another was related to the type strain of $S$. chromogenes and a third was allocated to a cluster containing a strain of $S$. epidermidis isolated from a UTI and two veterinary strains of $S$. intermedius. A further five isolates, including the type strain of $S$. saprophyticus (NCTC 7292), were unrelated to any of the other organisms tested.

The 21 isolates confirmed as $S$. saprophyticus by API STAPH were randomly distributed among the 34 clusters of staphylococci determined by Clustan analysis. Representatives of these 21 isolates were not only grouped within the six clusters composed predominantly of strains of $S$. saprophyticus but were also associated with representative strains of other staphylococcal species (table XII).

The percentage positive results for each individual species in all of the tests was calculated. Representative results are shown in table XIII. These results show that the tests most discriminative for differentiating $S$. saprophyticus from the other species of staphylococci are: resistance to novobiocin $5 \mu \mathrm{g} / \mathrm{ml}$; absence of phosphatase activity; aerobic fermentation of xylitol and maltose to produce acid; the utilisation of 2-acetamido-2deoxy-D-glucopyranoside methylumbelliferone; $\alpha$ L-arabinopyranoside-7-amino-4-methylumbelliferone; $\beta$-D-fucopyranoside-7-amino-4-methylumbelliferone; H-orn-7-amino-4-methylcoumarin; and pyr-7-amino-4-methylcoumarin. None of the tests performed in this study could alone adequately speciate $S$. saprophyticus.

\section{Discussion}

In the routine clinical laboratory, a urinary tract pathogen characterised as a CNS is presumptively identified as $S$. saprophyticus on the basis of a single test-resistance to novobiocin. Other CNS, e.g., $S$. cohnii $S$. sciuri and $S$. xylosus, are also resistant to novobiocin at a concentration of $5 \mu \mathrm{g} / \mathrm{ml}$ ( $\mathrm{Yu}$ and Washington, 1985). These organisms are not commonly associated with UTI, although $S$. xylosus has been implicated in urinary infections in men (Tselenis-Kotsowilis et al., 1982). Also, antibiotic resistance in staphylococci is often encoded on plasmids and, as such, is available for inter-species transfer. Care should be taken, therefore, to ensure accurate speciation.

The API STAPH identification system, which does not incorporate a test for resistance to novobiocin, determined that three of the 36 clinical isolates studied were strains of $S$. epidermidis, $S$. hominis and $S$. simulans $(\mathrm{p} \geq 97 \%)$. In the extended survey, which incorporated carbohydrate fermentation, antibiotic sensitivity (including resistance to novobiocin), and fluorogenic substrate tests, individual presumptive $S$. saprophyticus isolates possessed similar reaction profiles to the species $S$. cohnii, $S$. chromogenes, $S$. intermedius, $S$. xylosus and $S$. simulans. Conversely, other species of staphylococci shared phenotypic similarities with $S$. saprophyticus. This finding illustrates the heterogeneity of novobiocin-resistant CNS associated with UTI.

Nine clinical isolates tested by API STAPH possessed profile numbers which were equally 
Table IV. The fermentation of selected carbohydrates by clinical isolates of $S$. saprophyticus

\begin{tabular}{|c|c|c|c|c|c|c|c|c|c|c|c|c|c|c|c|c|c|c|c|c|c|}
\hline \multirow[b]{2}{*}{$\begin{array}{l}\text { Strain } \\
\text { no. }\end{array}$} & \multicolumn{21}{|c|}{ Fermentation (acid production, + ) of } \\
\hline & 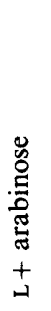 & 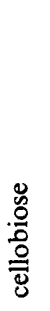 & 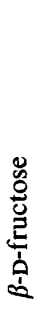 & 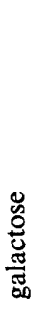 & 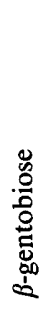 & 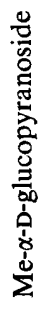 & 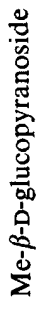 & $\begin{array}{l}\mathscr{ஜ} \\
\stackrel{0}{0} \\
: 00\end{array}$ & $\begin{array}{l}\overline{0} \\
. \overline{0} \\
. \\
.\end{array}$ & 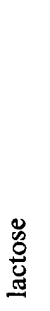 & 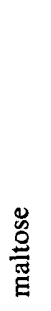 & 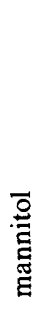 & 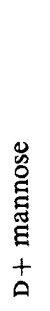 & 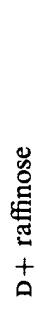 & 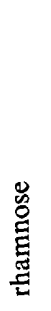 & $\frac{\bar{D}}{\stackrel{0}{0}}$ & 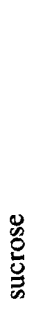 & 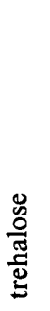 & 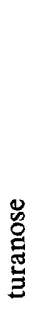 & $\frac{\overline{0}}{\frac{3}{2}}$ & $\frac{\ddot{y}}{x_{x}}$ \\
\hline L002 & - & - & + & - & - & - & + & + & - & - & - & + & - & - & - & - & + & + & - & - & - \\
\hline L003 & - & - & + & - & - & - & - & + & - & - & - & + & - & - & - & - & - & + & - & + & - \\
\hline L004 & - & - & + & - & - & - & + & + & - & - & - & + & - & - & - & - & + & + & - & - & - \\
\hline L005 & - & - & + & - & - & - & + & + & - & - & - & + & - & - & - & - & + & + & - & - & - \\
\hline L006 & - & - & + & - & - & - & + & + & - & - & - & + & - & - & - & - & + & + & - & - & - \\
\hline L007 & - & - & + & - & - & - & - & + & - & - & + & + & - & - & - & - & - & + & - & + & - \\
\hline L010 & - & - & + & - & - & - & + & + & - & - & + & + & - & - & - & - & + & + & - & + & - \\
\hline L011 & - & - & + & - & - & - & + & + & - & - & - & + & - & - & - & - & + & + & - & + & - \\
\hline L014 & - & - & + & - & - & - & + & + & - & + & - & - & - & - & - & - & + & + & - & - & - \\
\hline L016 & - & - & + & - & - & - & + & + & - & - & - & + & - & - & - & - & + & + & - & - & - \\
\hline L017 & - & - & + & - & - & - & - & + & - & - & + & + & - & - & - & - & + & + & - & + & + \\
\hline L018 & - & - & + & - & - & - & - & + & - & + & + & - & - & - & - & - & + & + & + & + & + \\
\hline L019 & - & - & + & - & - & - & + & + & - & - & + & - & - & - & - & - & + & - & - & - & - \\
\hline L020 & - & - & + & - & + & - & - & + & - & + & + & + & - & - & - & - & + & + & - & + & - \\
\hline L021 & - & - & + & - & - & - & + & + & - & - & - & - & - & - & - & - & + & + & - & - & - \\
\hline L022 & - & - & + & - & - & - & + & + & - & - & - & - & - & - & - & - & + & + & - & - & - \\
\hline L024 & - & - & + & + & - & - & + & + & - & + & - & - & + & - & - & - & + & + & - & - & - \\
\hline L025 & - & - & + & - & - & - & + & + & - & - & - & + & - & - & - & - & + & + & - & - & - \\
\hline L026 & - & - & + & - & - & - & + & + & - & - & - & + & - & - & - & - & + & + & - & - & - \\
\hline L027 & - & - & + & - & - & - & + & + & - & + & + & + & - & - & - & - & + & + & - & - & + \\
\hline L029 & - & - & + & - & - & - & + & + & - & + & + & + & - & - & - & - & + & + & - & - & - \\
\hline L030 & - & - & + & - & + & - & - & + & - & + & + & + & - & - & - & - & + & + & - & - & + \\
\hline L032 & - & - & + & - & + & - & + & + & - & - & + & + & - & - & - & - & + & + & - & + & - \\
\hline L034 & + & - & + & - & - & - & - & + & - & + & + & + & - & - & + & - & + & + & - & + & - \\
\hline L035 & - & - & + & - & - & - & - & + & - & - & + & + & - & - & - & - & - & + & - & + & - \\
\hline L036 & - & - & + & - & - & - & - & + & - & + & + & + & - & - & - & - & + & + & - & + & - \\
\hline L037 & - & - & + & - & - & - & + & + & - & - & + & + & - & - & - & - & + & + & - & - & - \\
\hline L039 & - & - & + & - & - & - & + & + & - & + & + & + & - & - & - & - & + & + & - & + & - \\
\hline L040 & - & - & + & - & - & - & + & + & - & - & - & + & - & - & - & - & + & + & - & - & - \\
\hline L041 & - & - & + & - & + & - & + & + & - & - & - & + & - & - & - & - & + & + & - & - & - \\
\hline L042 & - & - & + & - & - & - & + & + & - & - & - & + & - & - & - & - & + & + & - & - & - \\
\hline L043 & - & - & + & - & - & - & + & + & - & - & - & + & - & - & - & - & + & + & - & - & - \\
\hline L044 & - & - & + & - & - & - & + & + & - & + & + & + & - & - & - & - & + & + & + & + & - \\
\hline L056 & - & - & + & - & - & - & - & + & + & + & + & + & + & + & - & + & + & + & - & - & - \\
\hline L059 & - & - & - & - & - & - & - & + & - & - & - & - & - & - & - & - & - & - & - & - & - \\
\hline L031 & - & - & + & - & + & - & + & + & - & + & + & + & - & - & - & - & + & + & - & + & - \\
\hline
\end{tabular}

representative of more than one species of staphylococcus. In this situation the API STAPH identification system recommends testing for resistance to novobiocin, thereby enabling differentiation of $S$. saprophyticus from other CNS. There is, therefore, total reliance on a single characteristic for speciation since confirmation of the identity of $S$. saprophyticus is based upon the same test as the initial presumptive identification.

Conventional identification tests such as resistance to novobiocin and phosphatase activity were found to be particularly useful in differentiating $S$. saprophyticus from $S$. epidermidis. Additional tests which may prove useful in the differentiation of $S$. 
Table V. The fermentation of selected carbohydrates by reference strains of staphylococci

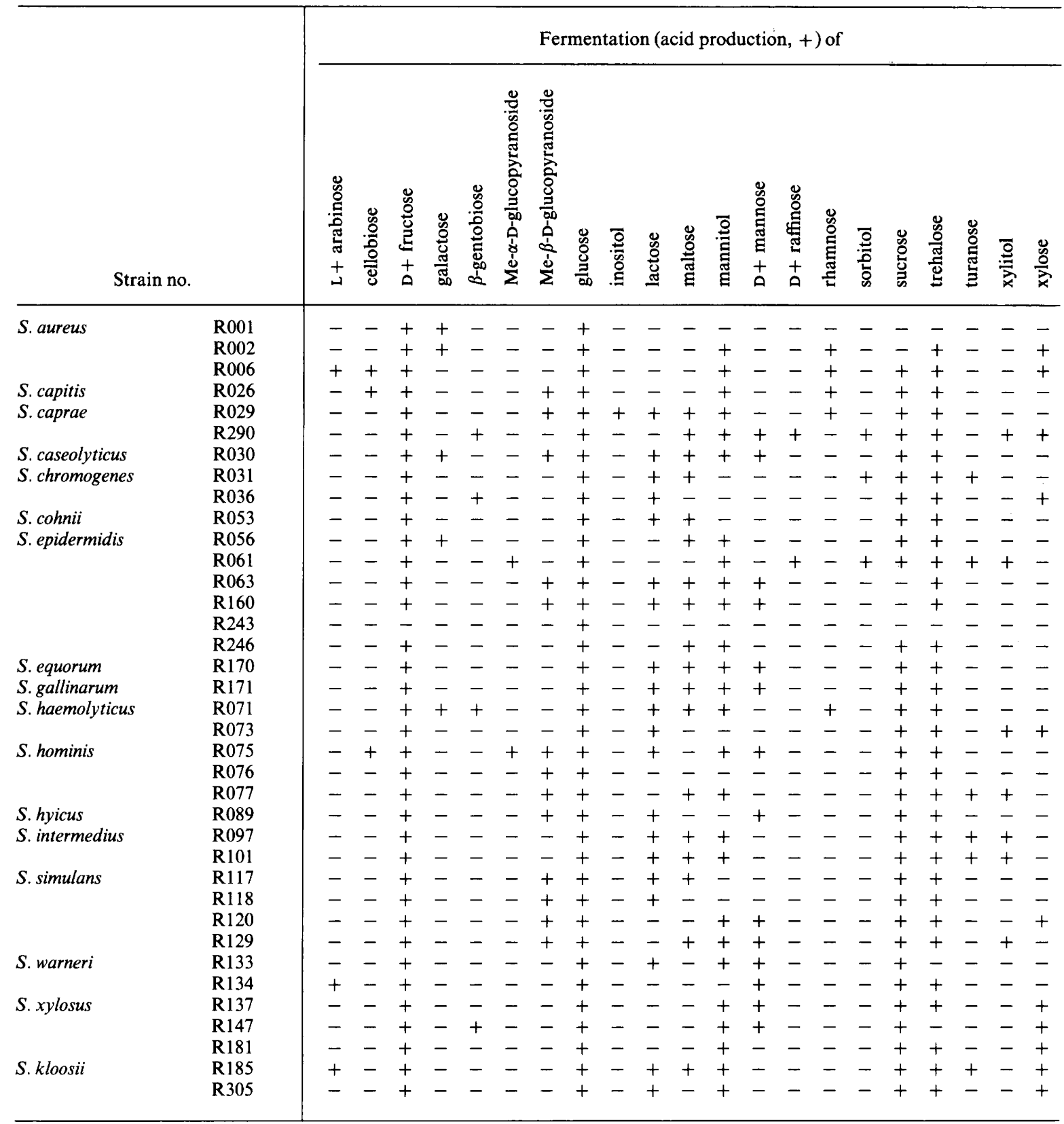

saprophyticus from other CNS have been determined as a result of the present work. These include the ability to cleave $\beta$-D-fucopyranoside from fluorogenic 7-amino-4-methylumbelliferone, a property attributable to more strains of $S$. saprophyticus than of other staphylococcal species studied. Unlike the Kloos and Schleifer system, these tests are rapid and easy, require only standard laboratory equipment, and so are useful in the routine clinical laboratory. The fluorogenic substrate tests are particularly rapid and yield results within $1 \mathrm{~h}$ of testing.

These findings suggest that tests for resistance to novobiocin and absence of phosphatase activity should both be performed in the routine clinical microbiology laboratory to differentiate $S$. sapro- 
Table VI. The antibiotic resistance pattern, DNAase and phosphatase activities of $S$. saprophyticus

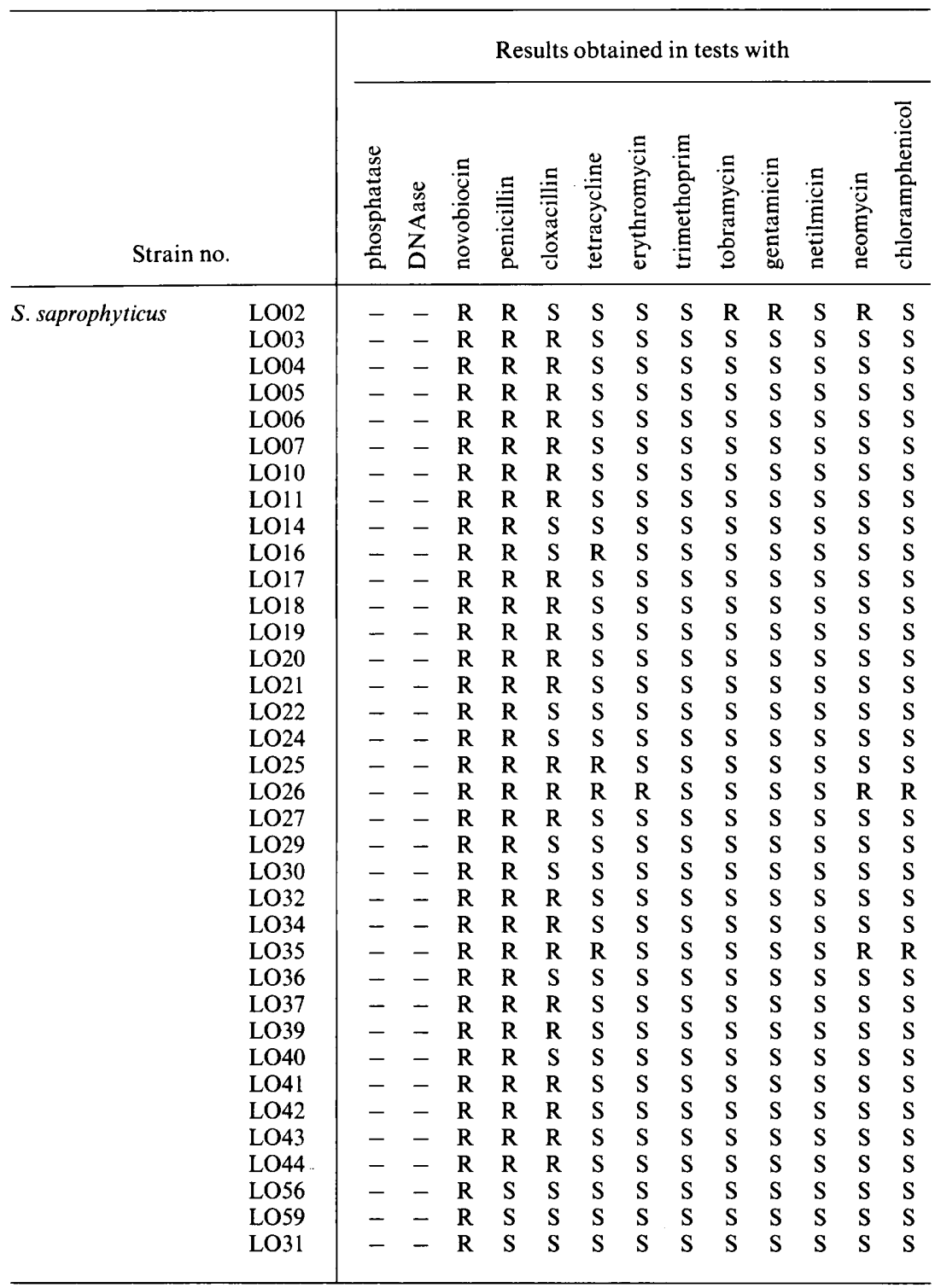

+ , Positive result DNAase, phosphatase activity; $R$, resistance to the antibiotic; $S$, sensitivity to the antibiotic.

phyticus from $S$. epidermidis. In studies requiring accurate speciation of $S$. saprophyticus, for example the role of S. saprophyticus in the pathogenesis of UTI in young women, further tests should be performed as suggested in this present study.
This work was presented in part at the 156th meeting of the Pathological Society of Great Britain and Ireland held in January 1988. The authors acknowledge the advice of Dr M. Goodfellow, Department of Microbiology, University of Newcastle upon Tyne. 
Table VII. The antibiotic resistance pattern, DNAase and phosphatase activities of reference strains of staphylococci

\begin{tabular}{|c|c|c|c|c|c|c|c|c|c|c|c|c|c|c|}
\hline \multirow{2}{*}{\multicolumn{2}{|c|}{ Strain no. }} & \multicolumn{13}{|c|}{ Results obtained in tests with } \\
\hline & & 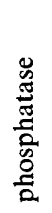 & 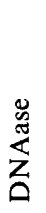 & $\begin{array}{l}\text { ! } \\
0 \\
0 \\
0 \\
0 \\
0 \\
0\end{array}$ & 志 & 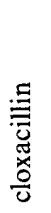 & 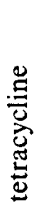 & 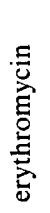 & 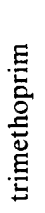 & 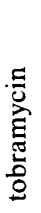 & 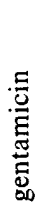 & 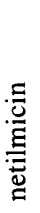 & 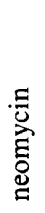 & 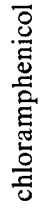 \\
\hline \multirow[t]{3}{*}{ S. aureus } & R001 & + & + & $\mathrm{S}$ & $\mathrm{R}$ & $\mathbf{S}$ & $\mathbf{S}$ & S & $\mathbf{S}$ & $\mathbf{S}$ & $\mathbf{S}$ & $\mathbf{S}$ & $\mathrm{S}$ & $\mathbf{S}$ \\
\hline & $\mathrm{R} 002$ & + & + & $S$ & $\mathrm{R}$ & $S$ & $\mathrm{~S}$ & $\mathrm{~S}$ & $\mathrm{~S}$ & $\mathbf{S}$ & $S$ & $\mathrm{~S}$ & $\mathrm{~S}$ & $\mathrm{~S}$ \\
\hline & R006 & + & + & $\mathrm{S}$ & $\mathbf{R}$ & $\mathbf{S}$ & $\mathbf{R}$ & S & $\mathrm{S}$ & $\mathrm{S}$ & $\mathbf{S}$ & $\mathrm{S}$ & $\mathrm{S}$ & $\mathrm{S}$ \\
\hline S. capitis & R026 & + & - & $\mathbf{S}$ & $\mathrm{S}$ & S & $\mathrm{S}$ & S & $\mathbf{S}$ & $\mathrm{S}$ & $\mathbf{S}$ & $\mathbf{S}$ & $\mathrm{S}$ & $\mathrm{S}$ \\
\hline \multirow[t]{2}{*}{ S. caprae } & R029 & - & + & $\mathrm{S}$ & $\mathrm{S}$ & S & $\mathrm{S}$ & $S$ & $\mathbf{S}$ & $\mathbf{S}$ & $\mathbf{S}$ & $\mathbf{S}$ & $\mathrm{S}$ & $\mathrm{S}$ \\
\hline & R290 & - & - & $\mathbf{S}$ & $\mathbf{S}$ & S & $\mathrm{S}$ & S & $\mathbf{S}$ & $\mathbf{S}$ & $\mathbf{S}$ & $\mathbf{S}$ & $\mathrm{S}$ & $\mathrm{S}$ \\
\hline S. caseolyticus & R030 & - & - & $\mathrm{S}$ & $\mathrm{S}$ & $\mathrm{S}$ & $\mathbf{S}$ & S & $\mathbf{S}$ & $\mathbf{S}$ & $\mathbf{S}$ & $\mathbf{S}$ & $\mathrm{S}$ & $\mathrm{S}$ \\
\hline \multirow[t]{2}{*}{ S. chromogenes } & R031 & + & + & $\mathrm{S}$ & $\mathrm{S}$ & $\mathbf{S}$ & $\mathbf{S}$ & S & $\mathrm{S}$ & $\mathbf{S}$ & $\mathbf{S}$ & $\mathrm{S}$ & $\mathrm{S}$ & $\mathrm{S}$ \\
\hline & R036 & + & + & $\mathbf{S}$ & $\mathbf{S}$ & $S$ & $\mathbf{S}$ & S & $\mathbf{S}$ & $\mathrm{S}$ & $\mathrm{S}$ & $\mathbf{S}$ & $\mathrm{S}$ & $\mathrm{S}$ \\
\hline S. cohnii & $\mathrm{R} 053$ & + & - & $\mathbf{R}$ & $\mathbf{R}$ & $\mathbf{R}$ & $\mathbf{S}$ & $\mathbf{S}$ & $\mathbf{S}$ & $\mathbf{S}$ & $\mathbf{S}$ & $\mathbf{S}$ & $\mathrm{S}$ & $\mathrm{S}$ \\
\hline \multirow[t]{6}{*}{ S. epidermidis } & R056 & + & - & $\mathbf{S}$ & $\mathrm{R}$ & $\mathrm{S}$ & $\mathbf{R}$ & S & $\mathrm{S}$ & $\mathrm{S}$ & $\mathrm{S}$ & $\mathrm{S}$ & $\mathrm{S}$ & S \\
\hline & R061 & + & - & $\mathrm{S}$ & $\mathrm{R}$ & $\mathrm{S}$ & $\mathbf{R}$ & $S$ & $\mathrm{R}$ & $\mathbf{R}$ & $\mathrm{S}$ & $\mathbf{S}$ & $\mathrm{S}$ & S \\
\hline & R063 & + & - & $\mathrm{R}$ & $\mathbf{R}$ & $\mathbf{R}$ & $\mathbf{S}$ & $\mathbf{S}$ & $\mathbf{R}$ & $\mathbf{R}$ & $\mathbf{S}$ & $\mathbf{S}$ & $\mathrm{S}$ & $\mathrm{S}$ \\
\hline & $\mathrm{R} 160$ & + & - & $\mathrm{S}$ & $\mathrm{R}$ & $\mathrm{S}$ & $\mathbf{R}$ & S & $\mathrm{S}$ & $\mathbf{S}$ & $\mathbf{S}$ & $\mathrm{S}$ & $\mathrm{S}$ & $\mathrm{S}$ \\
\hline & $\mathrm{R} 243$ & + & - & $\mathbf{S}$ & $\mathbf{R}$ & $\mathbf{S}$ & $\mathbf{R}$ & $S$ & $\mathrm{~S}$ & $\mathrm{~S}$ & $\mathrm{~S}$ & $\mathrm{~S}$ & $\mathrm{~S}$ & $\mathrm{~S}$ \\
\hline & $\mathrm{R} 246$ & + & - & $\mathrm{S}$ & $\mathrm{S}$ & S & $\mathbf{R}$ & $\mathrm{S}$ & $\mathbf{S}$ & $\mathbf{S}$ & $\mathbf{S}$ & $\mathbf{S}$ & $\mathrm{S}$ & $\mathrm{S}$ \\
\hline S. equorum & R170 & + & - & $\mathbf{R}$ & $\mathbf{R}$ & $\mathbf{R}$ & $\mathbf{R}$ & $\mathbf{R}$ & $\mathbf{S}$ & $\mathbf{S}$ & $\mathbf{S}$ & $\mathbf{S}$ & $\mathrm{S}$ & S \\
\hline S. gallinarum & R171 & + & - & $\mathrm{R}$ & $\mathbf{R}$ & $\mathbf{R}$ & $\mathbf{R}$ & $\mathrm{S}$ & $\mathrm{S}$ & $\mathrm{S}$ & $\mathrm{S}$ & $\mathrm{S}$ & $\mathrm{S}$ & $\mathbf{R}$ \\
\hline \multirow[t]{2}{*}{ S. haemolyticus } & R071 & + & - & $\mathrm{R}$ & $\mathbf{R}$ & $\mathrm{S}$ & $\mathbf{R}$ & S & $\mathbf{R}$ & $\mathbf{R}$ & $\mathbf{R}$ & $\mathbf{R}$ & $\mathrm{S}$ & $\mathbf{R}$ \\
\hline & $\mathrm{R} 073$ & - & - & $\mathrm{S}$ & $\mathrm{R}$ & $S$ & $\mathbf{R}$ & S & $\mathbf{R}$ & $\mathbf{R}$ & $\mathbf{S}$ & $\mathbf{S}$ & $\mathbf{R}$ & $\mathrm{S}$ \\
\hline \multirow[t]{3}{*}{ S. hominis } & R075 & + & - & $\mathrm{S}$ & $\mathbf{S}$ & $\mathbf{R}$ & $\mathbf{S}$ & S & $\mathrm{S}$ & $\mathrm{S}$ & $\mathbf{S}$ & $S$ & $\mathbf{S}$ & $S$ \\
\hline & R076 & + & - & $\mathbf{R}$ & $\mathbf{S}$ & $\mathbf{S}$ & $\mathbf{S}$ & $\mathrm{S}$ & $\mathbf{S}$ & $\mathrm{S}$ & $\mathbf{S}$ & $\mathbf{S}$ & $\mathbf{R}$ & $\mathrm{S}$ \\
\hline & R077 & + & - & $\mathbf{R}$ & $\mathrm{S}$ & $\mathbf{R}$ & $\mathbf{S}$ & $\mathbf{S}$ & $\mathbf{S}$ & $\mathbf{S}$ & $\mathbf{S}$ & $\mathbf{S}$ & $\mathbf{R}$ & S \\
\hline S. hyicus & R089 & + & - & $\mathbf{S}$ & $\mathrm{S}$ & $\mathbf{R}$ & $\mathbf{R}$ & S & $\mathrm{S}$ & $\mathrm{S}$ & $\mathrm{S}$ & $\mathrm{S}$ & $\mathrm{S}$ & $\mathrm{R}$ \\
\hline \multirow[t]{2}{*}{ S. intermedius } & R097 & + & - & $\mathrm{S}$ & $\mathrm{S}$ & $\mathbf{R}$ & $\mathrm{S}$ & $\mathrm{S}$ & $\mathrm{S}$ & $\mathrm{S}$ & $\mathbf{S}$ & $\mathrm{S}$ & $\mathbf{S}$ & $\mathrm{S}$ \\
\hline & R101 & + & - & $\mathrm{S}$ & $\mathrm{S}$ & $S$ & $\mathrm{~S}$ & S & $S$ & $\mathbf{S}$ & $\mathrm{S}$ & $\mathrm{S}$ & $\mathbf{S}$ & $\mathbf{S}$ \\
\hline \multirow[t]{4}{*}{ S. simulans } & R117 & + & - & $\mathrm{R}$ & $\mathbf{R}$ & $\mathbf{R}$ & $\mathbf{S}$ & S & $\mathbf{R}$ & $\mathrm{S}$ & $S$ & $\mathbf{S}$ & $S$ & $\mathrm{R}$ \\
\hline & R118 & + & - & $\mathbf{R}$ & $\mathrm{S}$ & S & $\mathbf{S}$ & $\mathrm{S}$ & $\mathbf{S}$ & $\mathrm{S}$ & $\mathbf{S}$ & $\mathbf{S}$ & $\mathrm{S}$ & $\mathrm{S}$ \\
\hline & R 120 & - & - & $\mathrm{S}$ & $S$ & $\mathbf{R}$ & $\mathbf{S}$ & S & $\mathbf{S}$ & $\mathrm{S}$ & $\mathbf{S}$ & $\mathrm{S}$ & $\mathbf{S}$ & $\mathrm{S}$ \\
\hline & R128 & - & - & $\mathrm{R}$ & $\mathrm{S}$ & S & $\mathrm{S}$ & $\mathrm{R}$ & $\mathrm{S}$ & $\mathrm{S}$ & $\mathbf{S}$ & $\mathbf{S}$ & $\mathrm{S}$ & $\mathrm{S}$ \\
\hline \multirow[t]{2}{*}{ S. warneri } & R133 & - & - & $\mathbf{S}$ & $\mathbf{S}$ & $\mathbf{S}$ & $\mathrm{S}$ & S & $\mathbf{S}$ & $\mathbf{S}$ & $\mathbf{S}$ & $\mathbf{S}$ & $\mathrm{S}$ & S \\
\hline & R134 & + & - & $\mathbf{S}$ & $\mathbf{R}$ & $S$ & $\mathrm{~S}$ & $\mathrm{~S}$ & $\mathrm{~S}$ & $\mathbf{S}$ & $\mathbf{S}$ & $\mathrm{S}$ & $\mathrm{S}$ & $\mathrm{S}$ \\
\hline \multirow[t]{3}{*}{ S. xylosus } & R137 & + & - & $\mathbf{R}$ & $\mathbf{R}$ & $\mathbf{S}$ & $\mathbf{S}$ & S & $\mathbf{S}$ & $\mathbf{S}$ & $\mathbf{S}$ & $\mathbf{S}$ & $\mathbf{S}$ & $\mathrm{S}$ \\
\hline & R147 & + & - & $\mathbf{R}$ & $\mathbf{R}$ & $\mathbf{R}$ & $\mathbf{R}$ & $\mathbf{S}$ & $\mathbf{S}$ & $\mathbf{S}$ & $\mathbf{S}$ & $\mathbf{S}$ & $\mathrm{S}$ & $\mathbf{S}$ \\
\hline & R181 & + & - & $\mathrm{R}$ & $\mathbf{R}$ & $\mathrm{S}$ & $\mathrm{S}$ & $\mathrm{S}$ & $\mathrm{S}$ & $\mathrm{S}$ & $\mathrm{S}$ & $\mathrm{S}$ & $\mathrm{S}$ & $\mathrm{S}$ \\
\hline \multirow[t]{2}{*}{ S. kloosii } & R 185 & - & - & $\mathbf{R}$ & $\mathbf{R}$ & $S$ & $\mathrm{~S}$ & $\mathrm{~S}$ & $\mathrm{~S}$ & $\mathrm{~S}$ & $\mathbf{S}$ & $\mathrm{S}$ & $\mathrm{S}$ & $\mathrm{S}$ \\
\hline & R305 & - & - & $\mathbf{R}$ & $\mathbf{R}$ & $\mathbf{S}$ & $\mathrm{S}$ & $\mathrm{S}$ & $\mathbf{S}$ & $\mathbf{S}$ & $\mathbf{S}$ & $\mathbf{S}$ & $\mathbf{S}$ & S \\
\hline
\end{tabular}

+ , Positive DNAase, phosphatase activity; $R$, resistance to the antibiotic; $S$, sensitivity to the antibiotic. 
Table VIII. The utilisation of derivatives of 7-amino-4-methylumbelliferone by 36 strains of $S$. saprophyticus

\begin{tabular}{|c|c|c|c|c|c|c|c|c|c|c|c|c|c|c|c|c|c|c|c|c|c|}
\hline \multirow[b]{2}{*}{$\begin{array}{l}\text { Strain } \\
\text { no. }\end{array}$} & \multicolumn{21}{|c|}{ Derivatives metabolised } \\
\hline & 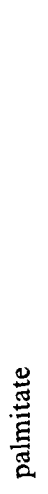 & 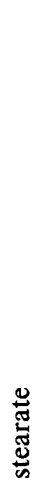 & 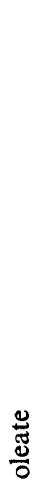 & 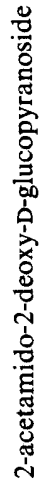 & 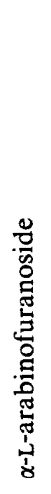 & 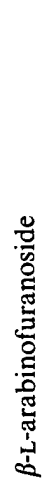 & 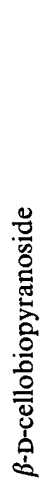 & 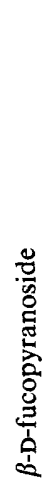 & 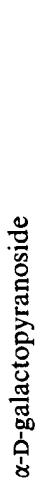 & 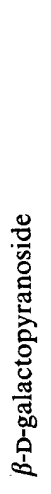 & 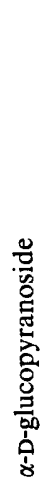 & 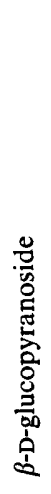 & 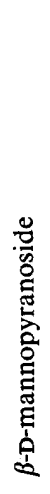 & 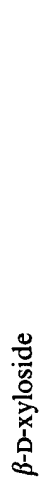 & 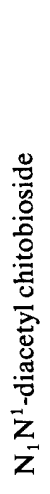 & 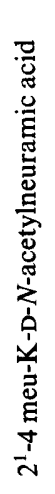 & 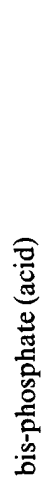 & 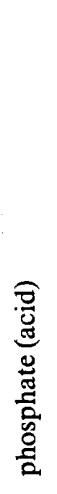 & 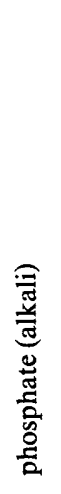 & 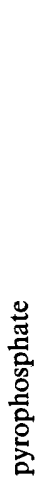 & 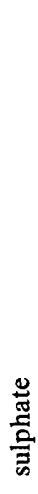 \\
\hline L002 & + & + & - & - & - & - & - & - & - & - & - & - & - & + & - & - & - & - & - & - & + \\
\hline L003 & + & + & + & - & - & - & - & - & - & - & - & + & - & - & - & - & + & + & - & - & + \\
\hline L004 & + & + & + & + & - & + & - & - & - & - & - & - & - & + & - & + & - & - & - & - & + \\
\hline L005 & + & - & - & - & - & - & - & - & - & - & - & - & - & - & - & - & + & - & + & + & - \\
\hline L006 & - & - & - & - & + & - & - & - & - & - & - & - & - & - & - & - & - & - & - & - & - \\
\hline L007 & - & - & - & - & - & - & - & - & - & - & - & - & - & - & - & - & + & - & + & - & - \\
\hline L010 & + & + & - & - & - & - & - & - & - & - & - & - & - & - & - & - & - & - & - & - & + \\
\hline L011 & + & + & - & + & - & + & - & - & - & - & - & - & - & - & - & - & - & - & - & - & + \\
\hline L014 & - & - & - & - & - & - & - & - & - & - & - & - & - & - & - & - & - & - & - & - & + \\
\hline L016 & + & + & - & + & - & - & - & - & - & - & - & - & - & - & - & - & - & - & - & - & + \\
\hline L017 & + & - & - & - & - & - & - & - & + & - & - & - & - & - & - & - & - & - & - & + & + \\
\hline L018 & + & + & + & + & - & + & - & - & - & - & - & - & - & - & - & - & + & - & + & - & - \\
\hline L019 & + & + & - & - & - & - & - & - & + & - & - & - & - & - & - & - & - & - & - & + & + \\
\hline L020 & + & + & - & + & - & + & - & - & - & - & - & - & - & + & - & - & - & - & - & + & + \\
\hline L021 & + & + & - & + & - & - & - & + & + & - & + & - & - & - & - & - & - & - & - & + & + \\
\hline L022 & + & - & - & - & - & - & - & - & - & - & - & + & - & - & - & - & + & - & + & - & + \\
\hline L024 & + & + & - & + & - & + & - & + & - & - & - & - & - & - & - & - & - & - & - & - & + \\
\hline L025 & + & + & - & - & - & - & - & + & + & - & - & - & - & - & - & - & - & - & - & + & + \\
\hline L026 & + & + & - & - & - & + & - & - & - & - & - & - & - & - & - & - & - & - & - & + & + \\
\hline L027 & + & + & - & + & - & - & - & - & + & - & + & - & - & - & - & - & - & - & - & - & + \\
\hline L029 & + & + & - & - & - & - & - & - & - & - & - & + & - & - & - & - & - & - & - & - & + \\
\hline L030 & - & - & - & - & - & - & + & - & - & - & - & + & - & - & - & - & - & - & - & + & + \\
\hline L032 & + & + & - & - & - & + & - & + & - & - & - & - & - & - & - & + & - & - & - & + & + \\
\hline L034 & + & + & - & - & - & - & - & - & - & - & - & + & - & - & - & - & + & + & - & - & + \\
\hline L035 & + & + & + & + & - & + & - & - & - & - & - & - & - & - & - & - & - & - & - & + & + \\
\hline L036 & + & + & - & + & - & + & - & + & + & - & + & - & - & - & - & - & - & - & - & - & + \\
\hline L037 & + & + & - & - & - & - & - & + & - & - & - & + & - & + & - & - & - & - & - & - & + \\
\hline L039 & + & - & - & - & - & + & - & - & - & - & + & + & - & + & - & - & + & - & + & + & + \\
\hline L040 & + & + & - & + & - & + & - & + & - & - & - & + & - & - & - & - & - & - & - & - & + \\
\hline L041 & + & + & - & - & - & - & - & + & - & - & - & - & - & - & - & - & - & - & - & - & + \\
\hline L042 & + & + & - & + & - & - & - & + & - & - & - & - & - & - & - & - & - & - & - & + & + \\
\hline L043 & + & - & - & - & - & - & - & - & + & - & - & - & + & - & - & - & - & + & - & + & + \\
\hline L044 & + & + & + & - & - & - & - & + & - & - & - & - & - & - & - & - & - & - & - & + & + \\
\hline L056 & + & + & - & - & - & - & - & - & - & - & - & - & - & - & - & - & - & - & - & + & + \\
\hline L059 & + & + & - & - & - & - & - & + & + & - & - & - & - & - & - & + & - & - & - & + & + \\
\hline L031 & + & - & - & - & - & - & - & + & - & - & - & - & - & - & - & - & + & - & + & + & - \\
\hline
\end{tabular}


Table IX. The utilisation of 7-amino-4-methylumbelliferone derivatives by reference strains of staphylococci

\begin{tabular}{|c|c|c|c|c|c|c|c|c|c|c|c|c|c|c|c|c|c|c|c|c|c|c|}
\hline Strain no. & & 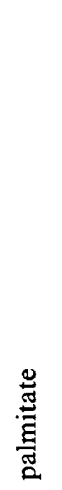 & 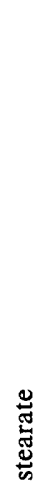 & $\begin{array}{l}\frac{ \pm}{ \pm} \\
\frac{\mathbb{J}}{0}\end{array}$ & 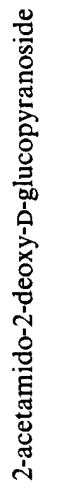 & 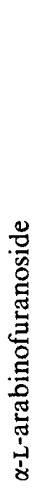 & 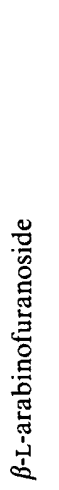 & 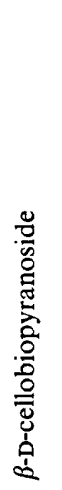 & 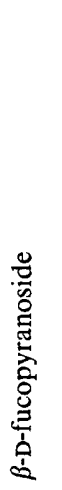 & 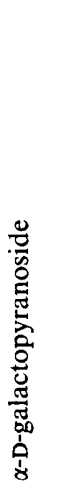 & 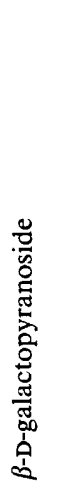 & 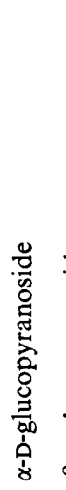 & 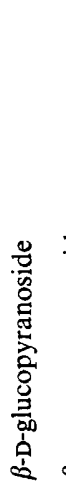 & 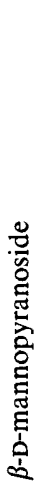 & 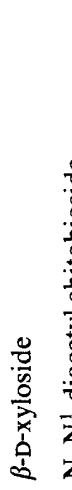 & 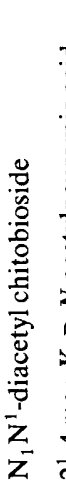 & 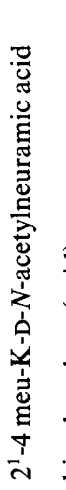 & 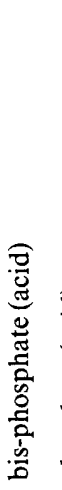 & 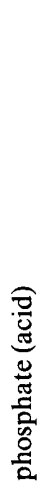 & 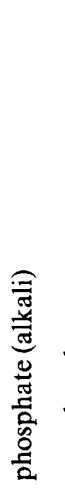 & 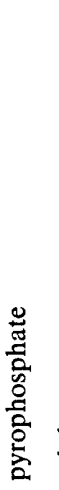 & 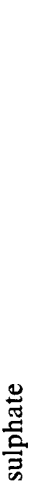 \\
\hline \multirow[t]{3}{*}{ S. aureus } & R001 & + & + & - & - & - & - & - & - & - & - & - & - & - & + & - & - & - & - & - & - & + \\
\hline & $\mathrm{R} 002$ & + & + & + & - & - & - & - & - & - & - & - & + & - & - & - & - & + & + & - & - & + \\
\hline & $\mathrm{R} 006$ & + & + & + & + & - & + & - & - & - & - & - & - & - & + & - & + & - & - & - & - & + \\
\hline S. capitis & R026 & + & - & - & - & - & - & - & - & - & - & - & - & - & - & - & - & + & - & + & + & - \\
\hline \multirow[t]{2}{*}{ S. caprae } & R029 & + & - & - & - & - & + & - & - & - & - & - & - & - & - & - & - & - & - & - & - & - \\
\hline & $\mathrm{R} 290$ & - & - & - & - & - & - & - & - & - & - & - & - & - & - & - & - & + & - & + & - & - \\
\hline S. caseolyticus & R030 & + & + & - & - & - & - & - & - & - & - & - & - & - & - & - & - & - & - & - & - & + \\
\hline \multirow{2}{*}{ S. chromogenes } & R031 & + & + & - & + & - & + & - & - & - & - & - & - & - & - & - & - & - & - & - & - & + \\
\hline & R036 & - & - & _- & - & - & - & - & - & - & - & - & - & - & - & - & - & - & - & - & - & + \\
\hline S. cohnii & R053 & + & + & - & + & - & - & - & - & - & - & - & - & - & - & - & - & - & - & - & - & + \\
\hline \multirow[t]{6}{*}{ S. epidermidis } & R056 & + & - & - & - & - & - & - & - & + & - & - & - & - & - & - & - & - & - & - & + & + \\
\hline & R061 & + & + & + & + & - & + & - & - & - & - & - & - & - & - & - & - & + & - & + & - & - \\
\hline & R063 & + & + & - & - & - & - & - & - & + & - & - & - & - & - & - & - & - & - & - & + & + \\
\hline & R 160 & + & + & - & + & - & + & - & - & - & - & - & - & - & + & - & - & - & - & - & + & + \\
\hline & R243 & + & + & - & + & - & - & - & + & + & - & + & - & - & - & - & - & - & - & - & + & + \\
\hline & R246 & + & - & - & - & - & - & - & - & - & - & - & + & - & - & - & - & + & - & + & - & + \\
\hline S. equorum & R 170 & + & + & - & + & - & + & - & + & - & - & - & - & - & - & - & - & - & - & - & - & + \\
\hline S. gallinarum & R171 & + & + & - & - & - & - & - & + & + & - & - & - & - & - & - & - & - & - & - & + & + \\
\hline \multirow[t]{2}{*}{ S. haemolyticus } & R071 & + & + & - & - & - & + & - & - & - & - & - & - & - & - & - & - & - & - & - & + & + \\
\hline & R073 & + & + & - & + & - & - & - & - & + & - & + & - & - & - & - & - & - & - & - & - & + \\
\hline \multirow[t]{3}{*}{ S. hominis } & R075 & + & + & - & - & - & - & - & - & - & - & - & + & - & - & - & - & - & - & - & - & + \\
\hline & R076 & - & - & - & - & - & - & + & - & - & - & - & + & - & - & - & - & - & - & - & + & + \\
\hline & R077 & + & + & - & - & - & + & - & + & - & - & - & - & - & - & - & + & - & - & - & + & + \\
\hline S. hyicus & R089 & + & + & - & - & - & - & - & - & - & - & - & + & - & - & - & - & + & + & - & - & + \\
\hline \multirow[t]{2}{*}{ S. intermedius } & R097 & + & + & + & + & - & + & - & - & - & - & - & - & - & - & - & - & - & - & - & + & + \\
\hline & R10l & + & + & - & + & - & + & - & + & + & - & + & - & - & - & - & - & - & - & - & - & + \\
\hline \multirow[t]{4}{*}{ S. simulans } & R117 & + & + & - & - & - & - & - & + & - & - & - & + & - & + & - & - & - & - & - & - & + \\
\hline & R118 & + & - & - & - & - & + & - & - & - & - & + & + & - & + & - & - & + & - & + & + & + \\
\hline & R120 & + & + & - & + & - & + & - & + & - & - & - & + & - & - & - & - & - & - & - & - & + \\
\hline & R129 & + & + & - & - & - & - & - & + & - & - & - & - & - & - & - & - & - & - & - & - & + \\
\hline \multirow[t]{2}{*}{ S. warneri } & R133 & + & + & - & + & - & - & - & + & - & - & - & - & - & - & - & - & - & - & - & + & + \\
\hline & R 134 & + & - & - & - & - & - & - & - & + & - & - & - & + & - & - & - & - & + & - & + & + \\
\hline \multirow[t]{3}{*}{ S. xylosus } & R137 & + & + & + & - & - & - & - & + & - & - & - & - & - & - & - & - & - & - & - & + & + \\
\hline & R147 & + & + & - & - & - & - & - & - & - & - & - & - & - & - & - & - & - & - & - & + & + \\
\hline & R181 & + & + & - & - & - & - & - & + & + & - & - & - & - & - & - & + & - & - & - & + & + \\
\hline \multirow{2}{*}{ S. kloosii } & R185 & + & - & - & - & - & - & - & + & - & - & - & - & - & - & - & - & + & - & + & + & - \\
\hline & R305 & + & + & - & + & - & + & - & + & + & - & + & + & - & + & - & + & - & + & - & + & + \\
\hline
\end{tabular}


Table X. The utilisation of 7-amino-4-methylcoumarin (AMC)-derivatives by $S$. saprophyticus

\begin{tabular}{|c|c|c|c|c|c|c|c|c|c|c|c|c|c|c|c|c|c|c|c|c|c|}
\hline \multirow[b]{2}{*}{ Strain no. } & \multicolumn{21}{|c|}{ Derivatives metabolised } \\
\hline & 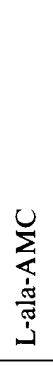 & 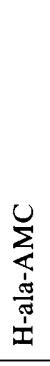 & 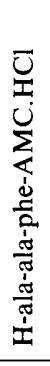 & 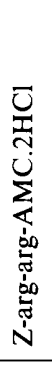 & 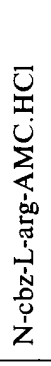 & 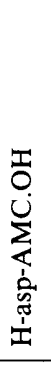 & 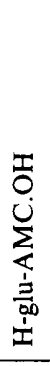 & 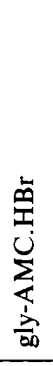 & 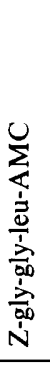 & 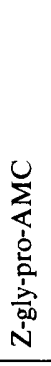 & 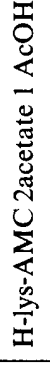 & 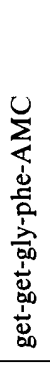 & 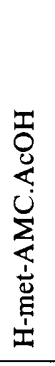 & 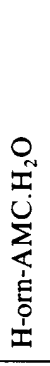 & 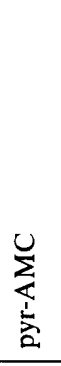 & 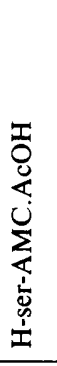 & $\sum_{\substack{U \\
⿱ 亠 䒑}}$ & 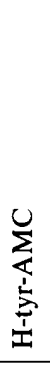 & 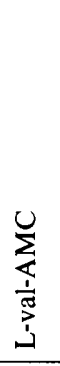 & 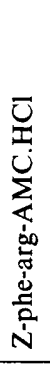 & 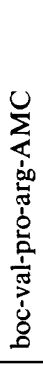 \\
\hline L002 & + & - & - & + & - & - & + & - & - & - & - & - & + & + & - & + & - & + & + & - & - \\
\hline L003 & - & - & - & - & - & - & - & - & - & - & - & - & - & - & - & + & - & - & - & - & - \\
\hline L004 & - & - & - & - & - & - & - & - & - & - & - & - & - & - & - & + & - & + & - & - & - \\
\hline L005 & - & - & - & - & - & - & - & - & - & - & - & - & - & - & + & - & - & - & - & - & - \\
\hline L006 & - & - & - & - & - & - & - & - & - & - & - & - & - & - & - & + & + & + & - & - & - \\
\hline L007 & + & - & - & - & - & - & - & - & - & - & - & - & - & - & - & + & - & - & - & - & - \\
\hline L010 & + & - & - & - & - & - & - & - & - & - & - & - & - & + & - & - & - & - & - & - & - \\
\hline L011 & - & - & - & - & - & - & - & - & - & - & - & - & - & - & - & + & - & - & - & - & - \\
\hline L014 & - & - & - & - & - & - & - & - & - & - & - & - & - & - & - & + & - & - & - & - & - \\
\hline L016 & - & - & - & + & - & - & + & - & - & - & - & - & - & + & + & + & + & - & - & - & - \\
\hline L017 & + & - & - & - & - & - & - & + & - & - & - & - & - & - & - & + & - & - & - & - & - \\
\hline L018 & + & - & - & - & - & - & - & - & - & - & - & - & - & - & - & - & - & - & - & - & - \\
\hline L019 & - & - & - & - & - & - & - & - & - & - & - & - & - & - & - & + & - & - & - & - & - \\
\hline L020 & + & - & - & + & - & - & - & - & - & - & - & - & - & + & - & - & - & - & - & - & - \\
\hline L021 & - & - & - & - & - & - & - & - & - & - & - & - & - & - & - & + & - & - & - & - & - \\
\hline L022 & + & - & - & + & - & - & + & - & - & - & - & - & + & + & - & + & - & - & - & - & - \\
\hline L024 & - & - & - & - & - & - & - & - & - & - & - & - & - & - & - & - & - & - & - & - & - \\
\hline L025 & + & - & - & - & - & - & - & - & - & - & - & - & - & + & - & - & - & - & - & - & - \\
\hline L026 & + & - & - & - & - & - & - & - & - & - & - & - & - & + & - & + & - & - & + & - & - \\
\hline L027 & - & - & + & - & - & - & - & - & - & - & - & - & - & - & + & - & - & - & - & - & - \\
\hline L029 & - & - & - & - & - & - & - & - & - & - & - & - & - & - & - & - & - & - & - & - & - \\
\hline L030 & - & - & - & - & - & - & - & - & - & - & - & - & - & - & + & - & - & - & - & - & - \\
\hline L032 & + & - & - & - & - & - & - & - & - & - & - & - & - & - & + & + & - & - & - & - & - \\
\hline L034 & + & - & - & - & - & - & - & - & - & - & - & - & - & - & + & - & - & + & - & - & - \\
\hline L035 & + & - & - & - & - & - & - & - & - & - & - & - & - & - & + & - & - & + & - & - & - \\
\hline L036 & - & - & + & - & - & - & - & - & - & - & - & - & - & - & + & - & - & - & - & - & - \\
\hline L037 & - & - & - & - & - & - & - & - & - & - & - & - & - & - & + & - & - & - & - & - & - \\
\hline L039 & - & - & - & - & - & - & - & - & - & - & - & - & + & - & + & - & - & - & - & - & - \\
\hline L040 & - & - & + & - & - & - & - & - & - & - & - & - & + & - & + & - & - & - & - & - & - \\
\hline L041 & - & - & - & - & - & - & - & - & - & - & - & - & + & - & + & - & - & - & - & - & - \\
\hline L042 & - & - & + & - & - & - & - & - & - & - & - & - & + & - & + & - & - & - & - & - & - \\
\hline L043 & - & - & - & - & - & - & - & - & - & - & - & - & - & - & + & - & - & - & - & - & - \\
\hline L044 & - & - & + & - & - & - & - & - & - & - & - & - & - & - & + & - & - & - & - & - & - \\
\hline L056 & + & - & - & - & - & - & - & + & - & - & - & + & + & - & + & - & - & - & - & - & + \\
\hline L059 & - & - & - & - & - & - & - & - & - & - & - & - & - & - & - & - & - & - & - & - & - \\
\hline L031 & - & - & + & - & - & - & - & - & - & - & - & - & - & - & + & - & - & - & - & - & - \\
\hline
\end{tabular}


Table XI. The utilisation of 7-amino-4-methylcoumarin (AMC) derivatives by reference strains of staphylococci

\begin{tabular}{|c|c|c|c|c|c|c|c|c|c|c|c|c|c|c|c|c|c|c|c|c|c|c|}
\hline \multirow[b]{2}{*}{ Strain no. } & & \multicolumn{21}{|c|}{ Derivatives metabolised } \\
\hline & & 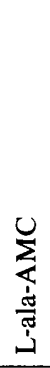 & 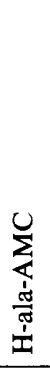 & 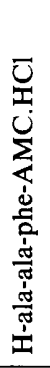 & 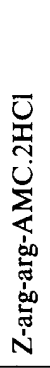 & 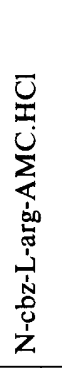 & 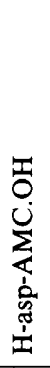 & 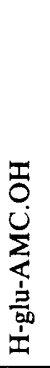 & 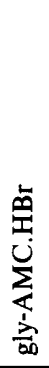 & 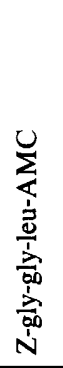 & 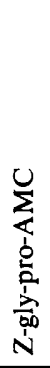 & 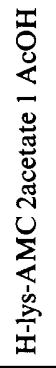 & 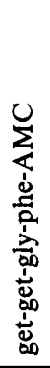 & 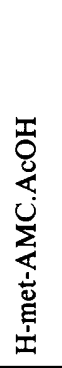 & 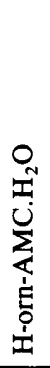 & 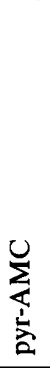 & 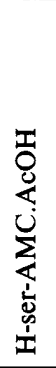 & 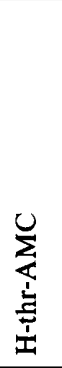 & 岕 & 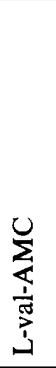 & 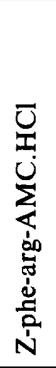 & 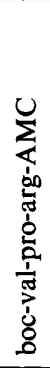 \\
\hline \multirow[t]{3}{*}{ S. aureus } & R001 & - & - & - & - & - & - & - & - & - & - & + & - & + & - & - & + & - & - & - & - & - \\
\hline & R002 & + & - & + & - & - & - & - & - & - & - & + & + & + & - & + & - & - & - & - & - & + \\
\hline & R006 & + & - & - & - & - & - & - & - & - & - & - & - & - & - & + & - & - & - & - & - & - \\
\hline S. capitis & R026 & + & - & - & - & - & - & - & + & - & - & + & - & + & - & + & - & - & - & - & - & + \\
\hline \multirow[t]{2}{*}{ S. caprae } & R029 & + & - & + & + & - & - & - & - & - & - & - & - & - & - & + & - & - & + & + & - & - \\
\hline & R290 & - & - & - & - & - & - & - & - & - & - & - & - & - & - & + & - & - & - & - & - & - \\
\hline S. caseolyticus & R030 & + & - & - & - & - & - & - & - & - & - & - & - & - & + & + & - & - & - & - & - & - \\
\hline \multirow[t]{2}{*}{ S. chromogenes } & R031 & - & - & - & - & - & - & - & - & - & - & + & - & - & + & - & - & - & - & - & - & - \\
\hline & R036 & - & - & - & - & - & - & - & - & - & - & - & - & - & - & + & - & - & - & - & - & - \\
\hline S. cohnii & R053 & + & - & - & - & - & + & - & + & - & + & - & - & - & - & - & - & - & - & - & - & - \\
\hline \multirow[t]{6}{*}{ S. epidermidis } & R056 & + & - & + & + & - & - & - & - & - & - & + & - & + & - & - & - & - & - & - & - & - \\
\hline & R061 & - & - & - & - & - & - & - & - & - & - & - & - & - & - & + & + & - & + & - & - & - \\
\hline & R063 & - & - & - & - & - & + & - & - & - & - & - & - & - & - & + & - & - & - & - & - & - \\
\hline & R160 & - & - & - & - & - & - & - & - & - & - & + & - & + & - & - & - & - & - & - & - & - \\
\hline & R243 & - & - & - & - & - & - & - & - & - & - & - & - & - & - & + & - & - & - & - & - & - \\
\hline & R246 & - & - & - & - & - & - & - & - & - & - & - & - & - & + & - & - & - & + & - & - & - \\
\hline S. equorum & R170 & - & - & + & - & - & - & - & - & - & - & - & - & - & - & - & - & - & - & - & - & - \\
\hline S. gallinarum & R171 & - & - & - & - & - & - & - & - & - & - & - & - & - & - & + & - & - & - & - & - & - \\
\hline \multirow[t]{2}{*}{ S. haemolyticus } & R071 & + & - & + & - & - & + & - & + & - & - & - & - & - & - & - & - & - & - & - & - & - \\
\hline & R073 & + & - & + & - & - & + & - & + & - & - & + & - & + & - & + & - & - & - & - & - & - \\
\hline \multirow[t]{3}{*}{ S. hominis } & R075 & - & - & + & - & - & - & - & - & - & - & + & - & + & - & + & + & - & + & - & - & - \\
\hline & R076 & - & - & - & - & - & - & - & - & - & - & - & - & - & - & + & - & - & - & - & - & - \\
\hline & R077 & + & - & - & - & - & - & + & - & - & - & + & - & + & - & - & - & - & - & - & - & - \\
\hline S. hyicus & R089 & - & - & - & + & - & - & - & - & - & - & - & - & - & + & + & + & + & - & - & - & + \\
\hline \multirow[t]{2}{*}{ S. intermedius } & R097 & - & - & - & - & - & - & - & - & - & - & - & - & - & - & + & - & - & - & - & - & - \\
\hline & R107 & - & - & - & - & - & - & - & - & - & - & - & - & - & - & + & - & - & - & - & - & - \\
\hline \multirow[t]{4}{*}{ S. simulans } & R117 & - & - & - & - & - & - & - & - & - & - & - & - & - & - & + & - & - & - & - & - & - \\
\hline & R118 & - & - & - & - & - & - & - & - & - & - & - & - & - & - & - & - & - & - & - & - & - \\
\hline & R120 & + & - & + & - & - & - & - & - & - & - & - & - & + & - & + & - & - & - & - & - & - \\
\hline & R129 & + & - & + & - & - & - & - & - & - & - & - & - & + & - & + & - & - & - & - & - & - \\
\hline \multirow{2}{*}{ S. warneri } & R133 & + & - & + & - & - & - & - & - & - & - & - & - & + & - & + & - & - & - & - & - & - \\
\hline & R134 & + & - & - & - & - & - & - & + & - & - & + & - & + & - & + & - & - & - & - & - & - \\
\hline \multirow[t]{3}{*}{ S. xylosus } & R137 & - & - & - & - & - & - & - & - & - & - & - & - & - & - & - & - & - & - & - & - & - \\
\hline & R147 & + & - & - & - & - & - & - & - & - & - & - & - & - & - & - & - & - & - & - & - & - \\
\hline & R181 & - & - & - & - & - & - & - & - & - & - & - & - & - & - & + & - & - & - & - & - & - \\
\hline \multirow{2}{*}{ S. kloosii } & R185 & - & - & - & - & - & - & - & - & - & - & - & - & - & - & + & - & - & - & - & - & - \\
\hline & R305 & + & - & - & - & - & - & - & - & - & - & - & - & - & - & - & + & - & - & - & - & - \\
\hline
\end{tabular}


Percentage similarity

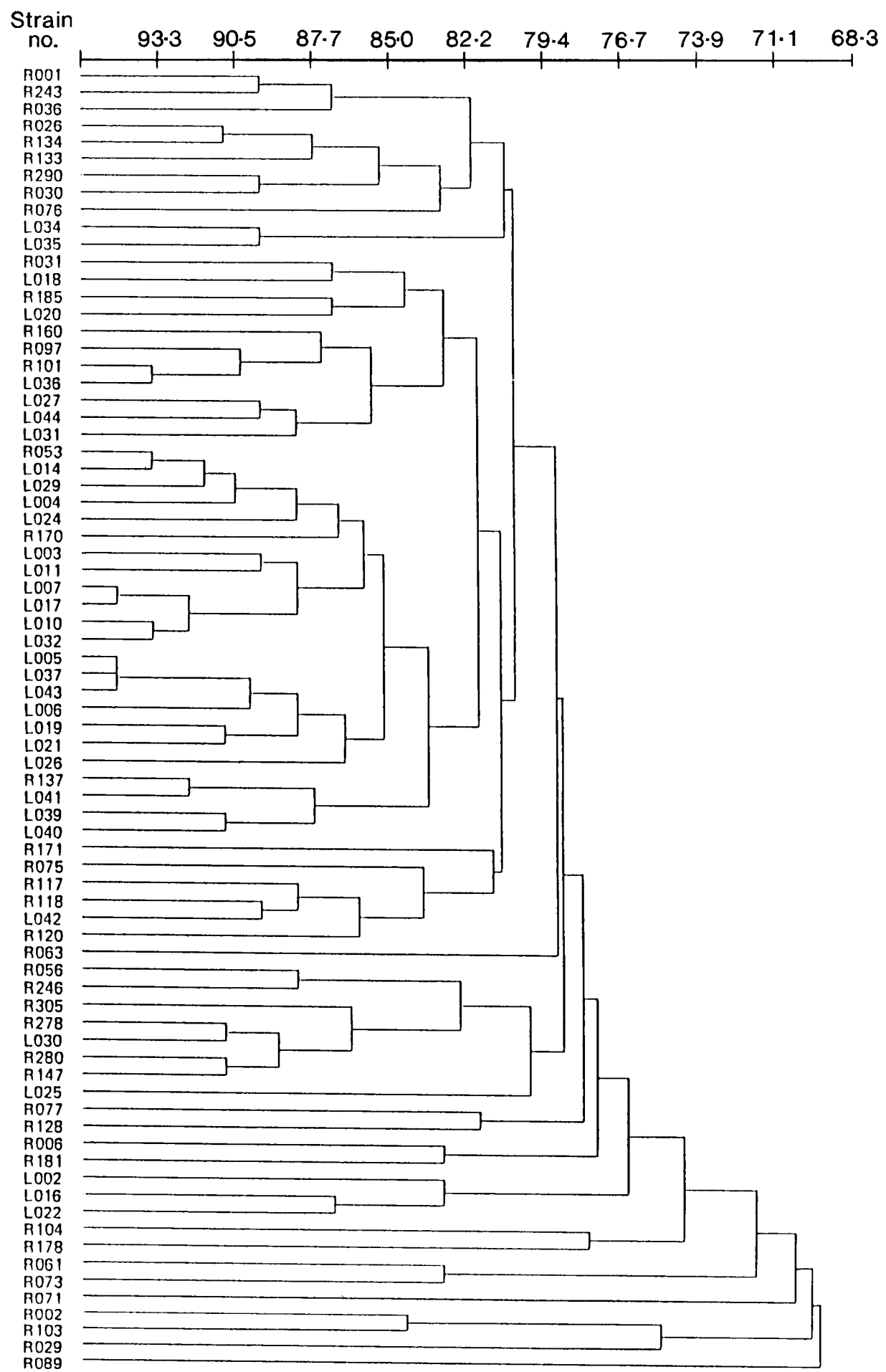

Figure. Dendrogram produced by Clustan analysis illustrating the percentage similarity of the test strains. 
Table XII. The 34 distinct clusters of related organisms obtained by Clustan analysis based upon staphylococcal phenotypic characteristics. When more than one strain of a particular species was investigated the number is shown in brackets. The cluster numbers represent the similarity of species within each cluster, consecutive groups being more closely related.

\section{S. aureus \\ $S$. epidermidis \\ S. chromogenes}

2. S. capitis

S. warneri

3. S. caprae

S. caseolyticus

4. S. hominis

5. S. saprophyticus $(2 / 1 \dagger)$

6. S. chromogenes

7. S. kloosi

S. saprophyticus $(1 / 1 \dagger)$

8. S. epidermidis

$S$. intermedius (2)

$S$. saprophyticus $(1 / 1 \dagger)$

9. S. saprophyticus $(3 / 1 \dagger)$

10. S. saprophyticus $(10 / 8) \dagger$

S. cohnii

$S$. equorum
11. S. saprophyticus $(7 / 4 \dagger)$

12. S. saprophyticus $(3 / 2 \dagger)$

S. xylosus

13. S. gallinarum

14. S. hominis

15. S. simulans

S. saprophyticus $(1 / 1 \dagger)$

16. S. epidermidis

17. S. kloosi

18. S. saprophyticus $(3 / 10 \dagger)$

S. xylosus

19. S. saprophyticus $(2 / 2 \dagger)$

20. S. hominis

21. S. simulans

22. S. aureus
23. S. xylosus

24. S. saprophyticus $(1 / 1 \dagger)$

25. S. saprophyticus $(2 / 0 \dagger)$

26. S. saprophyticus

27. S. saprophyticus*

28. S. epidermidis

29. S. haemolyticus

30. S. haemolyticus

31. S. aureus

32. S. lentus

33. S. lentus

34. S. hyicus

* Type strain of $S$. saprophyticus (NCTC 7292).

$\dagger$ Number of $S$. saprophyticus strains with identification confirmed by API STAPH. 
Table XIII. Percentage of strains of staphylococcal species able to ferment carbohydrates, utilise fluorogenic substrates and resistant to novobiocin $5 \mu \mathrm{g} / \mathrm{ml}$. Results for the 36 presumptive $S$. saprophyticus strains identified in the clinical laboratory and the 21 confirmed isolates are shown as original and confirmed respectively

\begin{tabular}{|c|c|c|c|c|c|c|c|c|c|c|}
\hline \multirow[b]{2}{*}{ Species } & \multirow{2}{*}{$\begin{array}{l}\text { Number of } \\
\text { strains tested }\end{array}$} & \multicolumn{9}{|c|}{ Percentage of strains that gave positive results in tests } \\
\hline & & M10 & M14 & M16 & $\mathrm{S} 10$ & $\mathbf{S} 22$ & A 18 & A19 & NOV & PHOS \\
\hline $\begin{array}{l}\text { S. saprophyticus } \\
\text { (original) }\end{array}$ & 36 & $58 \cdot 3$ & $58 \cdot 3$ & 18 & $33 \cdot 3$ & $58 \cdot 3$ & 18 & $58 \cdot 3$ & 100 & \\
\hline $\begin{array}{l}\text { S. saprophyticus } \\
\text { (confirmed) }\end{array}$ & 21 & $60 \cdot 8$ & $69 \cdot 5$ & $56 \cdot 5$ & $43 \cdot 3$ & 91 & $17 \cdot 3$ & $43 \cdot 4$ & 100 & \\
\hline S. aureus & 3 & $33 \cdot 3$ & $33 \cdot 3$ & & & & & 100 & & 100 \\
\hline S. capitis & 1 & & & & & & & & & 100 \\
\hline S. caprae & 2 & & 50 & & 50 & 100 & 100 & 100 & & 100 \\
\hline S. caseolyticus & 1 & & & & 100 & 100 & 100 & 100 & & \\
\hline S. chromogenes & 2 & 50 & 50 & & & 50 & 50 & 50 & & \\
\hline S. cohnii & 1 & 100 & & & & 100 & & & & \\
\hline S. epidermidis & 6 & & $33 \cdot 3$ & 16 & 16 & $66 \cdot 6$ & 16 & 50 & $33 \cdot 3$ & 100 \\
\hline S. equorum & 1 & 100 & 100 & & & 100 & & & 100 & 100 \\
\hline S. gallinarum & 1 & & & & & 100 & & 100 & 100 & \\
\hline S. haemolyticus & 2 & 50 & 50 & & 50 & 100 & & 100 & 50 & \\
\hline S. hominis & 3 & & $33 \cdot 3$ & & $33 \cdot 3$ & $33 \cdot 3$ & 100 & $33 \cdot 3$ & 100 & \\
\hline S. hyicus & 1 & & & & 100 & & & 100 & & \\
\hline S. intermedius & 2 & 100 & 100 & 50 & & 100 & & 100 & & 100 \\
\hline S. kloosii & 2 & & 50 & & & & & & 100 & \\
\hline S. simulans & 4 & 50 & 25 & 25 & 25 & & & 100 & 75 & 50 \\
\hline S. warneri & 2 & & & 50 & & & & & & \\
\hline S. xylosus & 3 & & $33 \cdot 3$ & 100 & & & & $66 \cdot 6$ & 100 & 100 \\
\hline
\end{tabular}

M10-utilisation of 2 acetamido-2-deoxy-D-glucopyranoside.

M14-utilisation of $\alpha$-L-arabinopyranoside-7-amino-4-methylumbelliferone.

M16 - utilisation of $\beta$-D-fucopyranoside-7-amino-4-methylumbelliferone.

S10-fermentation of xylitol.

S22-fermentation of maltose.

A18-utilisation of H-orn-7-amino-4-methylcoumarin.

A19-utilisation of pyr-7-amino-4-methylcoumarin

$\mathrm{NOV}$-resistance to novobiocin $5 \mu \mathrm{g} / \mathrm{ml}$.

PHOS-phosphatase activity.

\section{REFERENCES}

Bes M, Brun Y, Gayral J, Fleurette P, Laban P 1985 Improvement of "API STAPH" gallery and identification of new species of staphylococci. In: Jeljaszewicz J (ed) The staphylococci. Zentralblatt für Bakteriologie, Mikrobiologie und Hygiene Suppl 14. Gustav Fischer Verlag, Stuttgart.

Brun Y, Fleurette J, Forey F 1978 Micromethod for biochemical identification of coagulase negative staphylococci. Journal of Clinical Microbiology 8: 503-508.

Christensen G D, Parisi J T, Bisno A L, Simpson W A, Beachey E H 1983 Characterization of clinically significant strains of coagulase-negative staphylococci. Journal of Clinical Microbiology 18 : 258-269.

Hussain Z, Stoakes L, Stevens D L, Schieven B C, Lannigan R, Jones C 1986 Comparison of the MicroScan system with the "API Staph-Ident" system for species identification of coagulase-negative staphylococci. Journal of Clinical Microbiology 23 : 126-128.

Kloos W E, Schleifer K H 1975 Simplified scheme for routine identification of Staphylococcus species. Journal of Clinical Microbiology 1 : 82-87.

Lennox V A, Ackerman V P 1984 Biochemical identification of bacteria by replicator methods on agar plates. Pathology 16: 434-440.

Maskell R 1974 Importance of coagulase-negative staphylococci as pathogens in the urinary tract. Lancet 1: 1155-1158.

Morgan J W 1983 Abbreviated scheme for the presumptive identification of Staphylococcus saprophyticus from urine cultures. Journal of Clinical Microbiology 18: 1272-1274.

Nachaie M R 1983 Aspects of the classification and identification of medically important staphylococci. PhD Thesis, University of Newcastle-upon-Tyne.

Sneath P H A 1957 The application of computers to taxonomy. Journal of General Microbiology 17: 201-226.

Torres Pereira A 1962 Coagulase-negative strains of staphylococcus possessing antigen 51 as agents of urinary infection. Journal of Clinical Pathology 15 : 252-253.

Tselenis-Kotsowilis A D, Koliomichalis M P, Papavassiliou J T 1982 Acute pyelonephritis caused by Staphylococcus xylosus. Journal of Clinical Microbiology 16: 593-594.

Yu P K U, Washington J A 1985 Identification of staphylococci. In: Washington J A (ed) Laboratory procedures in clinical microbiology, 2nd edn. Springer Verlag, New York, pp 130131. 